\title{
Erratum to: Effect of extrusion process on microstructure and mechanical and corrosion properties of biodegradable $\mathrm{Mg}-5 \mathrm{Zn}-1.5 \mathrm{Y}$ magnesium alloy
}

Hassan Jafari ${ }^{\bowtie}$, Amir Houshang Mojiri Tehrani, and Mahsa Heydari

Materials Engineering Department, Faculty of Materials Engineering and Interdisciplinary Science, Shahid Rajaee Teacher Training University, $16785-136$

Tehran, Iran

Erratum to: International Journal of Minerals, Metallurgy and Materials

Volume 29, Number 3, March 2022, Page 490

https://doi.org/10.1007/s12613-021-2275-5

The original version of this article unfortunately contained a mistake. The author's name in the original version is incorrect, which is:

Hassan Jafari $^{\circledR}$, Amir Houshang Mojiri Tehrani, Mojiri Tehrani, and Mahsa Heydari

The correct version is given below:

Hassan Jafari $^{\bowtie}$, Amir Houshang Mojiri Tehrani, and Mahsa Heydari

The original version PDF file has been replaced by the corrected version PDF file.

The online version of the original article can be found at https://doi.org/10.1007/s12613-021-2275-5 